\title{
Comparative Study of Bit Error Rate in LDPC Based OFDM System over AWGN Channels
}

\author{
Vinayak Purohit ${ }^{1}$, Ramniwas Sahu ${ }^{2}$, Mayank Joshi ${ }^{3}$ \\ Department of Electronics \& Communication Engineering, Marudhar Engineering College, Bikaner
}

\begin{abstract}
Modern communication systems are increasingly adopting new Morden technologies like OFDM and LDPC for achieving high performance, low Bit Error Rate (BER) and high capacity. The OFDM communication is very much inspired from the channel frequencies over the network. In such a network some kind of orthogonal distortion occurs over the channel called Inter Carrier Interference.
\end{abstract}

Keywords- OFDM Techniques, Bit Error Rate, LDPC code, Inter Channel Interference, QAM Modulation, TDMA Techniques.

\section{INTRODUCTION}

In this chapter, we firstly review some important technologies that contribute to the modern digital communication system. These techniques, including the Orthogonal Frequency Division Multiplexing (OFDM) modulation and Low Density Parity Check (LDPC) code, will be the main topic of the dissertation. Following the problem statement, dissertation objective is introduced. Finally, the outline of the dissertation will be given.

OFDM represents a different system design approach. It can be thought of as a combination of modulation and multiple access schemes that segment a communication channel in such a way that many users can share it. Whereas TDMA segments are according to time and CDMA segments are according to spreading codes, OFDM segments are according to frequency. It is a technique that divides the spectrum into a number of equally spaced tones and carries a portion of a user's information on each tone. A tone can be thought of as a frequency, much in the same way that each key on a piano represents a unique frequency. OFDM can be viewed as a form of frequency division multiplexing (FDM), however, OFDM has an important special property that each tone is orthogonal with every other tone. FDM typically requires there to be frequency guard bands between the frequencies so that they do not interfere with each other. OFDM allows the spectrum of each tone to overlap, and because they are orthogonal, they do not interfere with each other. By allowing the tones to overlap, the overall amount of spectrum required is reduced.

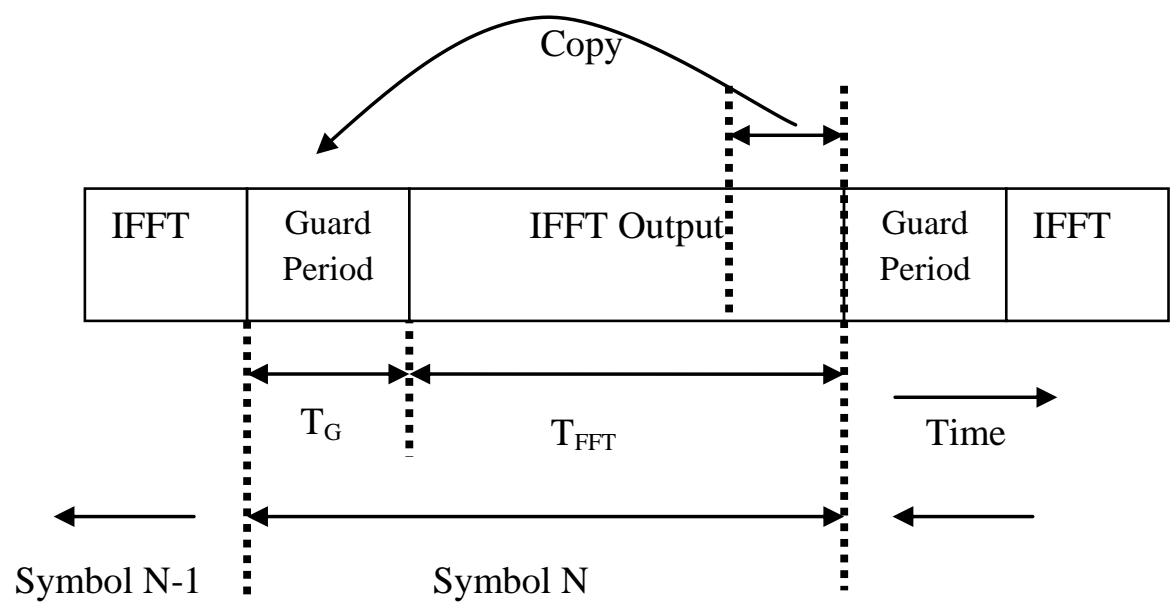

FIGURE 1: BLOCK DIAGRAM OF OFDM

\section{OFDM SYSTEM DESIGN}

The design of an OFDM system requires a tradeoff between various parameters as like in all communication system design. Usually, the input parameters to the design are the bit rate, available bandwidth and the maximum delay spread introduced by the channel. The design involves calculation of symbol duration, guard time, number of sub-carriers and the modulation and coding schemes among others. 


\subsection{OFDM System Design}

In this section, we consider a typical design problem with the following requirements.

- Bit Rate : 20Mbps

- Maximum Delay Spread : $\quad$ 200ns

- Available Bandwidth : $\quad 15 \mathrm{MHz}$

\subsection{Guard Time}

From the design considerations discussed previously, we see that a guard time of

$4 * 200 \mathrm{~ns}=800 \mathrm{~ns}$ is a reasonable choice.

\subsection{Modulation and Coding}

To transmit 20Mbps data, the number of bits to be transmitted in an OFDM symbol is $=20 \mathrm{Mbps} * 4.8 \mu \mathrm{s}=96 \mathrm{bits} / \mathrm{OFDM}$ symbol. Now, we can go for

- 16 - QAM with rate (1/2) coding for each sub-carrier so that there are 2 bits per symbol per sub carrier. Thus we see that 48 sub-carriers are required in this case.

- QPSK with rate (3/4) coding for each sub-carrier so that there are 1.5 bits per symbol per sub carrier. Thus in this case, we need 64 sub carriers.

But in the latter case, 64 sub-carriers require a bandwidth of $64 * 250 \mathrm{KHz}=16 \mathrm{MHz}$ which is greater than the available bandwidth of $15 \mathrm{MHz}$. Hence the first one is a good choice in the sense that it satisfies the constraints.

\section{LDPC CODES}

LDPC codes were originally introduced by Gallager in his doctoral dissertation [9]. Since the discovery of turbo codes in 1993 by Berrou and the rediscovery of LDPC codes by Mackay and Neal in 1995 [10], there has been renewed interest in turbo codes and LDPC codes because their bit error rate performance approaches asymptotically the Shannon limit [4]. Much research is devoted to characterizing the performance of LDPC codes and designing codes that have good performance. Commonly, a graph, the Tanner graph, is associated with the code and an important parameter affecting the performance of the code is the girth of its Tanner graph. The Tanner graph is a special type of graph, a bipartite graph, where the nodes divide into two disjoint classes with edges only between nodes in the two different classes.

\section{Simulations AND ReSUlts}

\subsection{OFDM Spectrum}

Orthogonal Frequency Division Multiplexing method has been used for spectrum generation. The main reason of using OFDM spectrum is that is distributes the data over large number of carriers that are spaced apart at different precise frequencies.

\subsection{AWGN Channel}

ADDITIVE WHITE GAUSSIAN noise (AWGN) channel is a universal channel model for analyzing modulation schemes. In this model, the channel does nothing but add a white Gaussian noise to the signal passing through it. This implies that the channel's amplitude frequency response is flat(thus with unlimited or infinite bandwidth) and phase frequency response is linear for all frequencies so that modulated signal pass through it without any amplitude loss and phase distortion of frequency components .

\subsection{BER Vs SNR of the LDPC Based OFDM system in AWGN channel Using QAM}

Using MATLAB Figure2 shows the BER Vs SNR of the LDPC Based OFDM system in AWGN channel using QAM modulation scheme. This figure shows the relationship between BER and SNR. The values of SNR are from $-30 \mathrm{db}$ to $0 \mathrm{db}$ and the scale of SNR is linear. The values of BER are from 0.1 to 0.5 and scale of BER is log. 


\section{TABLE 1}

SNR AND BER EVALUATION

\begin{tabular}{|c|c|c|}
\hline S. NO. & SNR & BER \\
\hline 1 & $-30 \mathrm{db}$ & 0.4767 \\
\hline 2 & $-25 \mathrm{db}$ & 0.4566 \\
\hline 3 & $-20 \mathrm{db}$ & 0.4221 \\
\hline 4 & $-15 \mathrm{db}$ & 0.3649 \\
\hline 5 & $-10 \mathrm{db}$ & 0.2672 \\
\hline 6 & $-5 \mathrm{db}$ & 0.1128 \\
\hline 7 & $0 \mathrm{db}$ & 0.0 \\
\hline
\end{tabular}

BER Vs SNR of LDPC based OFDM system in AWGN channel using 2QAM

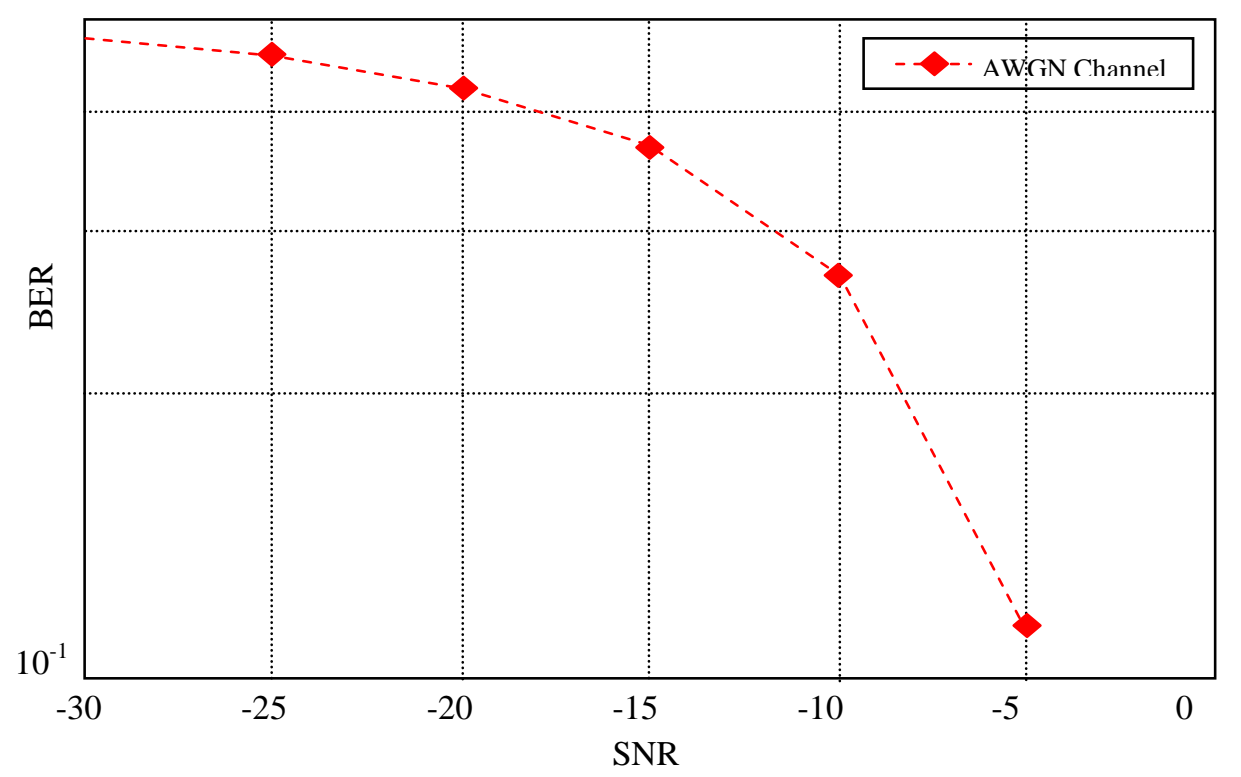

FIGURE 2: BER VS SNR OF THE LDPC BASED OFDM SYSTEM IN AWGN CHANNEL USING QAM

\section{REFERENCES}

[1]. Andrea Goldsmith, "Wireless Communications", Stanford UniversityCambridge University Press - 2005.

[2]. Louis Frenzel, "OFDM FAQ Tutorial," April 2009.

[3]. T. S. Rappaport, "Inter Carrier Interference Cancellation for OFDM Systems" EE-318 K-11, Wireless Communication, Springer, 2003.

[4]. C.E. Shannon, “A mathematical theory of communication”, Bell Syst. Tech.J., pp. 372-423, 1948.

[5]. Yao Xiao, "OFDM Multiplexing Modulation and ICI Cancellation,” B.S, Dalian University of Tech, 1998, May 2003.

[6]. Eric phillip, “Adaptive techniques for Multiuser OFDM,”LAWREYBE, Dec 2001.

[7]. S.Weinstein and P.Ebert, "Data transmission by frequency-division multiplexing using the discrete Fourier transform," IEEE Trans. Commun.,vol.-19, pp. 628-634, Oct.1971.

[8]. L.J. Cimini, "Analysis and Simulation of a Digital Mobile Channel Using Orthogonal Frequency Division Multiplexing”, IEEE Transactions on Communication no.7 July 1985.

[9]. R.G. Gallager,“Low-Density Parity Check Codes”. Cambridge, MA: MIT Press, 1963.

[10]. D.J.C. Mackay and R.M. Neal,“ Good codes based on very sparse matrices”, in Cryptography and Coding, 5th IMA Conference (Lecture Notes in Computer Science), C. Boyd, Ed. 1995, vol. 1025, pp. 110-111, 1995

[11]. Shu Lin and Costello, "Error Control Coding", Pearson-Prentice Hall, ISBN-13: 978-0130426727, 2004.

[12]. Angelos D. Liveris, ZixiangXiong and Costas N. Georghiades, "Compression of Binary Sources With Side Information at the Decoder Using LDPC Codes", IEEE Communications Letters, Vol. 6, NO.10, October 2002.

[13]. E.A. Lee and D.G. Messerschmitt,"Digital Communication”. Boston/Dordrecht/London: Kluwer, 1994.

[14]. Amendment: Physical Layer and Management Parameters for $10 \mathrm{~Gb} / \mathrm{s}$ Operation, Type 10GBASE-T, IEEE Draft P802.3an/D2.1.

[15]. T.J. Richardson, M.A. Shokrollahi, and R.L. Urbanke, "Design of capacity approaching irregular low-density parity-check codes", IEEE Trans. Inform. Theory, Vol. 47, No. 2, pp. 619-637, Feb. 2001. 